\title{
Surveillance of fetal lung lesions using the congenital pulmonary airway malformation volume ratio: natural history and outcomes ${ }^{\dagger}$
}

\author{
Catriona A. Macardle ${ }^{1}$, Stacey Ehrenberg-Buchner ${ }^{2}$, Ethan A. Smith³, Jonathan R. Dillman ${ }^{3}$, George B. Mychaliska ${ }^{2,4}$, \\ Marjorie C. Treadwell ${ }^{2}$ and Shaun M. Kunisaki $2,4 *$ \\ ${ }^{1}$ Department of Obstetrics and Gynecology, St. Joseph Mercy Health System, Ann Arbor, MI, USA \\ Department of Obstetrics and Gynecology, Division of Maternal Fetal Medicine, C.S. Mott Children's and Von Voigtlander Women's Hospital, \\ University of Michigan Health System, Ann Arbor, MI, USA \\ ${ }^{3}$ Department of Radiology, Section of Pediatric Radiology, C.S. Mott Children's and Von Voigtlander Women's Hospital, University of Michigan Health System, \\ Ann Arbor, MI, USA \\ ${ }^{4}$ Department of Surgery, Section of Pediatric Surgery, C.S. Mott Children's and Von Voigtlander Women's Hospital, University of Michigan Health System, \\ Ann Arbor, MI, USA \\ *Correspondence to: Shaun M. Kunisaki. E-mail: shaunkun@umich.edu \\ †2015 International Conference in Prenatal Diagnosis and Therapy, Washington, DC.
}

\begin{abstract}
Objectives The congenital pulmonary airway malformation volume ratio (CVR) is a widely used sonographic measure of relative mass size in fetuses with lung malformations. The purposes of this study were to examine serial CVR measurements to understand longitudinal growth patterns and to determine correlation with postnatal imaging.
\end{abstract}

Methods An institutional review board-approved retrospective review was performed on fetuses referred for an echogenic lung malformation between 2002 and 2014. For each fetus, the CVR was prospectively calculated using 2D ultrasound and followed with advancing gestation.

Results Based on 40 fetuses, the mean initial CVR was $0.51 \pm 0.07$ at $20.5 \pm 0.3$ weeks of gestation. The CVR increased after 24 weeks of gestation ( $p=0.0014$ ), peaking at a CVR of $0.96 \pm 0.11$ at $25.5 \pm 0.05$ weeks, followed by a significant decrease in the CVR to $0.43 \pm 0.07$ prior to term $(p<0.0001)$. However, approximately one third showed no appreciable increase in size. The mean CVR was significantly correlated with postnatal chest computed tomography (CT) size dimensions $(p=0.0032)$ and likelihood for lung resection $(p=0.0055)$.

Conclusions Fetal lung malformations tend to follow one of two distinct growth patterns, characterized by either (1) a maximal CVR between 25 and 26 weeks of gestation or (2) minimal change in relative growth. The mean CVR correlates with postnatal CT size and operative management. () 2015 John Wiley \& Sons, Ltd.

Funding sources: None

Conflicts of interest: None declared

\section{INTRODUCTION}

Over the last two decades, echogenic fetal lung malformations, which include congenital pulmonary airway malformations (CPAM) and bronchopulmonary sequestrations (BPS), have been diagnosed with greater frequency by ultrasound. ${ }^{1}$ Based on a recent study, the current incidence of lung malformations may be as high as one in 2000 to 2500 live births. ${ }^{2}$ The increased rate of detection is thought to be secondary to the widespread use of fetal ultrasound screening as well as ongoing improvements in the sensitivity of the anatomic survey, thereby resulting in the detection of smaller masses that do not cause mass effect. ${ }^{3}$ As a result, the risk of hydrops in fetuses with lung malformations is now lower than previously reported, ${ }^{4,5}$ and perinatal survival rates exceeding $95 \%$ are the norm. ${ }^{3,6,7}$
Previous work has shown that the congenital pulmonary airway malformation volume ratio (CVR), as measured by two-dimensional (2D) ultrasound, is a simple, useful way to assess relative mass volume and is an independent predictor of perinatal outcome in fetal lung malformations. ${ }^{3,8}$ To date, however, relative mass volume as a function of gestational age has not been well studied. ${ }^{9-11}$ In one report, initial size progression followed by regression occurs in approximately one third of all lesions. ${ }^{12}$ A study from the Children's Hospital of Philadelphia subsequently had similar findings based on both mass volume and CVR data and suggested a plateau in growth between 26 and 28 weeks of gestation. ${ }^{13}$ Unfortunately, these data were based on a sampling of different time points from different patients and not from serially following individual patients. ${ }^{14}$ Other studies from multiple centers 
have suggested that lung malformations may have markedly different growth patterns, ranging from significant increases in size throughout gestation, resulting in hydrops and need for fetal intervention, ${ }^{9,13,15}$ to spontaneous regression without growth in most lesions. ${ }^{6,10,16}$

In this study, we examined serial ultrasound data to determine the extent to which fetal lung malformations change in size throughout gestation. We hypothesized that the relative size of lung malformations, as measured by the CVR, might have predictable variation based on gestational age, which therefore has implications on prenatal management and postnatal outcome.

\section{METHODS}

This Health Insurance Portability and Accountability Actcompliant study was approved by the University of Michigan Institutional Review Board (\#51132). The medical records of consecutive fetuses referred for management of a lung malformation between January 2002 and March 2014 were retrospectively reviewed. Cases were ascertained from a prospectively maintained database at the Fetal Diagnosis and Treatment Center, a tertiary care referral center with comprehensive fetal intervention capabilities based at the C.S. Mott Children's and Von Voigtlander Women's Hospital, University of Michigan Health System. For a subset of these patients $(n=21)$, detailed sonographic data on lung malformations in relationship to respiratory morbidity have been previously reported. ${ }^{3}$

All fetuses underwent serial 2D sonograms (Phillips iU-22, Andover, MA, USA; General Electric Voluson 730, Waukesha, WI, USA; or ATL HDI 2000, Seattle, WA, USA) performed by a staff maternal-fetal medicine specialist. In each case, the presence of a systemic vascular supply to the lung lesion was assessed by color and power Doppler ultrasound. Fetal hydrops was defined as the presence of fluid in two or more spaces, including ascites, pleural effusion, pericardial effusion, or subcutaneous edema, usually with supporting echocardiographic evidence of cardiac dysfunction. ${ }^{17,18}$ The three-dimensional size of an echogenic lesion was prospectively measured (in $\mathrm{cm}$ ) to calculate a CVR as originally described by Crombleholme and colleagues. ${ }^{13}$ Briefly, the CVR was obtained by taking the product of the length, width, and depth of the mass, multiplying by a 0.523 correction factor (assuming its shape as a prolate ellipse), and then dividing by the head circumference to normalize for gestational age. In an effort to minimize any potential intra-operator and inter-operator variability over time, the maximal height of the lesion was first measured in the sagittal plane followed by measurement in the plane perpendicular to this axis. During the study period, the recommended frequency of subsequent ultrasound exams was not standardized but generally ranged between 1 and 4 weeks based on the most recent CVR measurement and the presence of hydrops.

Given the study goal of examining fetal lung lesion growth over time, fetuses had to satisfy specific inclusion criteria for further analysis of growth patterns, namely, at least one CVR measurement before 24.0 weeks with two or more subsequent scans between 24.0 weeks and delivery. Relative changes in the CVR over time were normalized based on the initial CVR $\left(\mathrm{CVR}_{0}\right)$. CVR measurements were also stratified based on early ( $<24.0$ weeks), middle (24.0-29.0 weeks), and late ( $>29.0$ weeks) gestation. Prenatal variables were analyzed and correlated with outcomes data. After 2009, maternal betamethasone was routinely given for microcystic lesions with CVRs greater than 1.6 with polyhydramnios or with any signs of hydrops or cardiac dysfunction based on data reported elsewhere. ${ }^{14}$

Postnatal assessment of lung malformations was performed by arterial phase contrast-enhanced chest computed tomography (CT) using either a 16 or 64 multi-detector scanner (GE Healthcare, Waukesha, WI, USA), in most cases electively between 2 and 4 months of age. ${ }^{19}$ Mass volume was measured by maximum size in three dimensions (anteroposterior, craniocaudal, and transverse) based on axial and multi-planar reconstructed images interpreted by board-certified, fellowship-trained pediatric radiologists, all of whom were blinded to prenatal studies, using a picture achieving and communication system workstation (Horizon Rad Workstation 11.9, McKesson, San Francisco, CA, USA). Operative resection was based on individual pediatric surgeon preference and parental concerns but was generally recommended for symptomatic lesions, macrocystic disease, asymptomatic microcystic CPAMs measuring greater than $2 \mathrm{~cm}$ in size, and asymptomatic intralobar masses associated with a systemic arterial vessel. ${ }^{20}$

All values were reported as the mean \pm standard error of the mean unless otherwise indicated. Statistical analyses were performed by the Wilcoxon matched-pairs signed rank test, repeated measures one-way analysis of variance, Mann-Whitney test, Spearman correlation, or second-degree polynomial regression analyses as appropriate using Prism 6.0 (GraphPad, La Jolla, CA, USA). Significance was defined as $p<0.05$.

\section{RESULTS}

\section{Baseline characteristics}

One hundred referrals for a fetal lung malformation were identified during the study period. The serial CVR measurements for these patients are shown in Figure 1. The mean CVR for the entire group was $0.72 \pm 0.08$ [median: 0.50, interquartile range (IQR): $0.26-0.86$ ]. There were 56 left-sided and 43 right-sided cases. One patient had bilateral lesions. A systemic feeding vessel arising from the thoracoabdominal aorta was identified in 23 fetuses. Twenty-six lesions were macrocystic, and 62 were associated with mediastinal shift on the initial scan.

Table 1 reveals the ultrasound characteristics of lesions based on whether inclusion criteria were met for longitudinal analysis. Among the 40 patients that met the inclusion criteria, the mean number of ultrasounds was $6.15 \pm 0.37$ (median: 5.5, IQR: 4.0-8.0). All had unilateral disease, composed of 21 (52.5\%) left-sided and 19 (47.5\%) right-sided masses. Thirty-three (82.5\%) lesions were microcystic, and seven (17.5\%) were macrocystic. Twenty-three (57.5\%) had associated mediastinal mass effect on initial exam. 


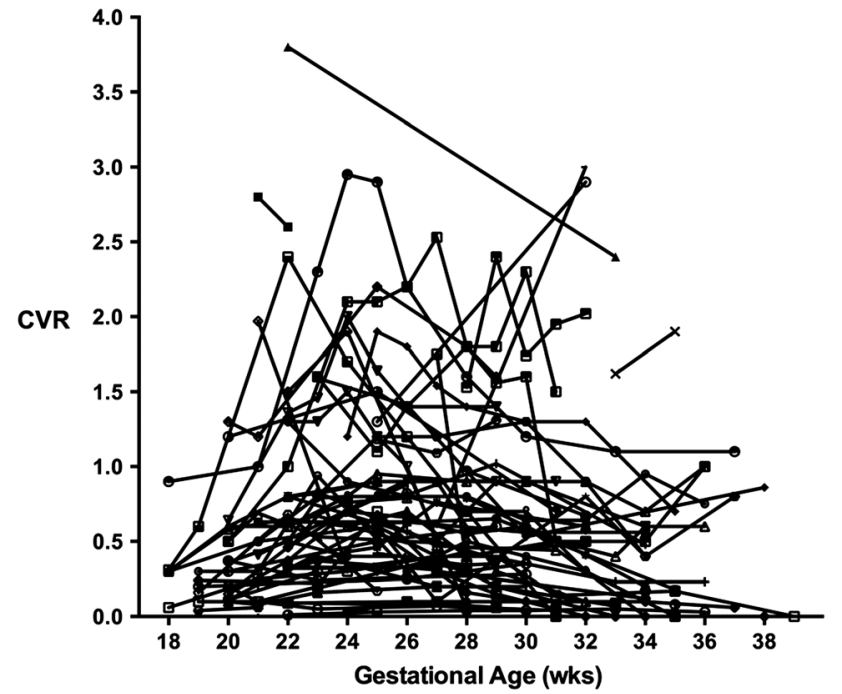

Figure 1 Line graph of serial congenital pulmonary airway malformation volume ratio (CVR) measurements in fetal lung malformations $(n=100)$. Each line represents the CVR measurements for an individual fetus throughout pregnancy

Eleven (27.5\%) had a systemic feeding vessel. Three (7.5\%) had concomitant extrapulmonary anomalies, including hydronephrosis $(n=2)$ and complex heart defect $(n=1)$. The $\mathrm{CVR}_{0}$ was $0.51 \pm 0.07$ (median: 0.31, IQR: 0.19-0.64). None of these patients had a $\mathrm{CVR}_{0}$ greater than 1.6. The mean CVR was $0.61 \pm 0.07$ (median: 0.5, IQR: 0.27-0.86).

Table 1 shows that, compared with fetuses excluded from the study, those included had a significantly greater number of ultrasound scans $(p<0.0001)$ and were diagnosed at an earlier gestational age $(p<0.0001)$. However, there were no significant differences based on laterality $(p=0.42)$, presence of a systemic feeding vessel ( $p=0.47)$, microcystic appearance $(p=0.11), \quad$ mediastinal shift $\quad(p=0.40), \mathrm{CVR}_{0} \quad(p=0.08)$, maximum CVR greater than $1.6(p=1.00)$, and mean CVR $(p=0.87)$. Whereas none of the included fetuses developed hydrops, four $(6.7 \%)$ in the excluded group developed hydrops. Because of small sample size, the difference in the rate of hydrops between the groups failed to meet statistical significance $(p=0.15)$.

Maternal betamethasone was administered to five included (13\%) and five excluded (8\%) patients $(p=0.52)$. All fetuses meeting inclusion criteria responded satisfactorily to corticosteroids as shown by a reduction in the maximum CVR of $2.14 \pm 0.29$ at $25.1 \pm 0.55$ weeks to a final CVR (mean, $1.16 \pm 0.17$ at $31.9 \pm 4.4$ weeks). Although there were no fetal lung resections or thoracoamniotic shunts performed in either group, three excluded fetuses underwent ex utero intrapartum treatment-to-resection compared with none in the included group $(p=0.27)$.

\section{Longitudinal growth patterns}

The CVR measurements for all patients meeting the inclusion criteria are shown in Figure $2 \mathrm{~A}$. The mean $\mathrm{CVR}_{0}$ was 0.51 \pm 0.07 at $20.5 \pm 0.3$ weeks of gestation (median weeks: 20.1, IQR: 19.6-21.9), and the mean final CVR was $0.41 \pm 0.07$ at $33.6 \pm 0.5$ weeks of gestation (median weeks: $33.7, \mathrm{IQR}$ : 31.1-36.3). There was no significant difference in the $\mathrm{CVR}_{0}$ compared with the final CVR ( $p=0.098)$.

The mean maximum CVR was $0.96 \pm 0.11$, which occurred at a mean of $25.5 \pm 0.05$ weeks of gestation (median weeks: 25.2, IQR: 23.5-27.2). Based on statistical modeling of serial CVR data using second-degree polynomial regression analysis $(R 2=0.1$, Figure $2 \mathrm{~B})$, the CVR peaks at $0.85 \pm 0.1$ at 26.1 weeks. However, there was growth variability among different malformations that was captured by two distinct growth profiles. Based on data normalized by the $\mathrm{CVR}_{0}$, the first group consisted of a small subset of malformations ( $n=13$, $32.5 \%)$ that either had minimal relative enlargement $(0-50 \%$

Table 1 Prenatal characteristics of lung malformations

\begin{tabular}{lccc} 
& & Adequate longitudinal ultrasound data? \\
\cline { 2 - 4 } Variable & Yes $(n=40)$ & No $(n=60)$ & $<$ \\
\hline Number of ultrasounds & $6.2 \pm 0.37$ & $2.7 \pm 0.25$ & $<0.0001$ \\
\hline Gestational age at initial scan, weeks & $20.5 \pm 0.28$ & $26.1 \pm 0.64$ & $<0.0001$ \\
\hline Left sided, $n(\%)$ & $21(53)$ & $36(61)$ & 0.42 \\
\hline Systemic feeder vessel, $n(\%)$ & $11(28)$ & $12(20)$ & 0.47 \\
\hline Microcystic, $n(\%)$ & $33(83)$ & $40(67)$ & 0.11 \\
\hline Mediastinal shift, $n(\%)$ & $23(58)$ & $40(67)$ & 0.40 \\
\hline Hydrops, $n(\%)$ & $0(0)$ & $4(7)$ & 0.15 \\
\hline CVR at initial scan & $0.51 \pm 0.07$ & $11(18)$ & 0.13 \\
\hline Maximum CVR $>1.6, n(\%)$ & $7(18)$ & $0.80 \pm 0.14$ & 1.00 \\
\hline Mean CVR & $0.61 \pm 0.07$ & $4(7)$ & 0.87 \\
\hline Other major anomalies, $n(\%)$ & $3(8)$ & $5(8)$ & 1.00 \\
\hline Maternal betamethasone, $n(\%)$ & $5(13)$ & $0(0)$ & 0.52 \\
\hline Fetal interventions, $n(\%)$ & $0(0)$ & 1.00 \\
\hline
\end{tabular}

CVR, congenital pulmonary airway malformation volume ratio. ${ }^{*} p<0.05$. 

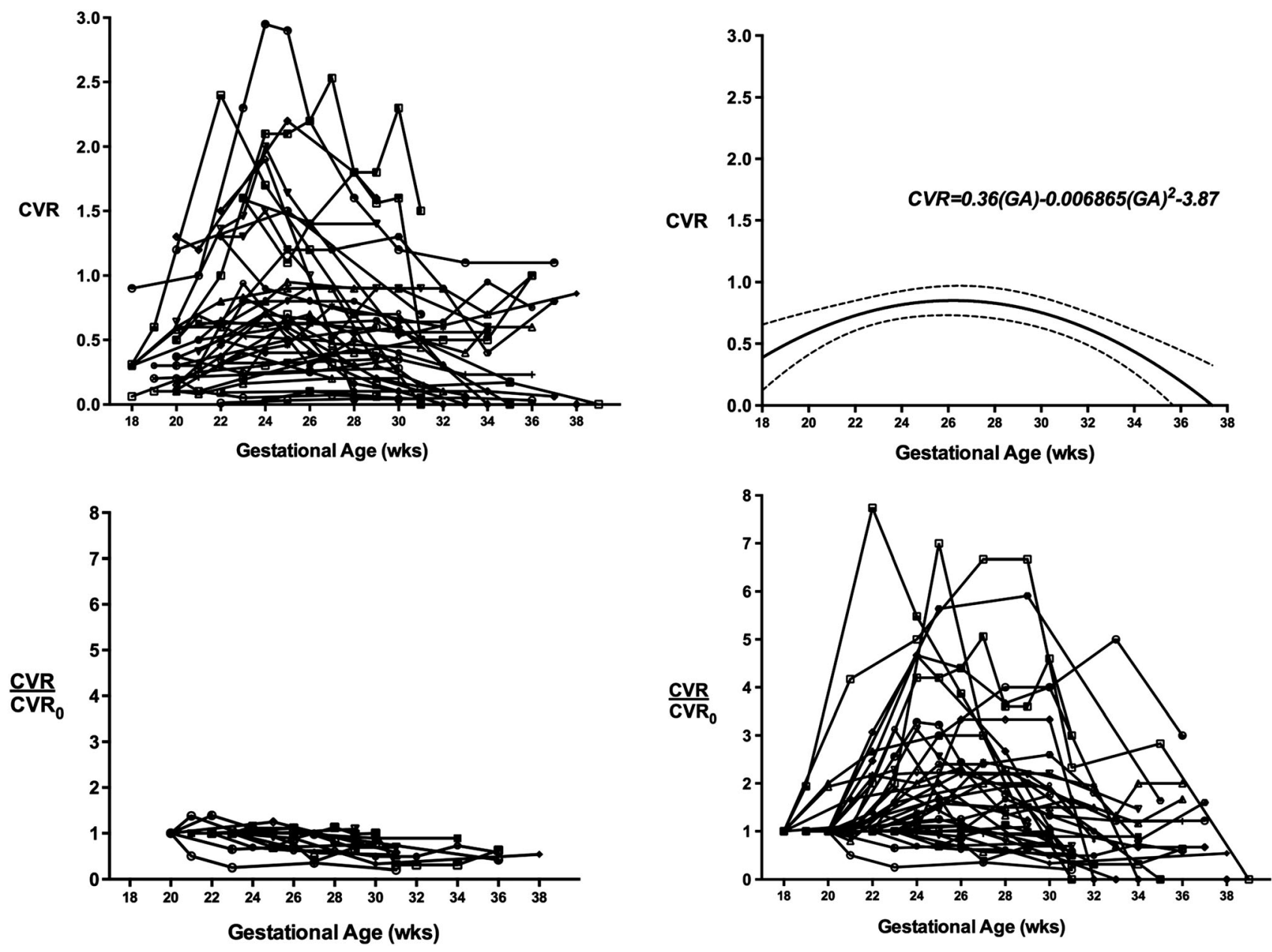

Figure 2 Serial congenital pulmonary airway malformation volume ratio (CVR) measurements of fetal lung malformations with adequate longitudinal follow-up data. (A) Line graph of CVR data ( $n=40$ lesions). (B) Statistical modeling of CVR data using second-degree polynomial regression analysis (solid curve) with 95\% confidence intervals (dotted curves). (C) Line graph depicting lack of relative CVR growth is a subgroup ( $n=13$ ) of lung lesions. $Y$-axis shows CVR data normalized by the initial CVR (CVRo). (D) Line graph depicting increased relative CVR growth before 30 weeks gestation in a subgroup $(n=27)$ of lung lesions. Y-axis shows CVR data normalized by the CVRo

increase) or a relative decrease in size throughout gestation (Figure 2C). The second group consisted of a larger subset of lesions $(n=27,67.5 \%)$ that tended to enlarge $(>50 \%$ size increase) between 24 and 29 weeks, after which a marked reduction in the CVR was observed up to the time of delivery (Figure 2D). This escalation followed by spontaneous regression in the CVR persisted even after exclusion of growth profiles that may have been altered by exposure to maternal betamethasone $(n=5)$.

After stratification of data points based on early, middle, and late gestational age cohorts, there was a significant increase from the $\mathrm{CVR}_{0}(0.51 \pm 0.07)$ compared with the middle gestation CVR $(0.74 \pm 0.09, p=0.0014$, Figure 3A) . Further univariate analysis based on various prenatal sonographic characteristics, including suspected CPAM, presence of a systemic feeding vessel, and microcystic disease, also revealed a significant tendency towards an increase in absolute CVR measurements between 24 and 29 weeks of gestation (Figure 3B-C).

In all fetuses followed longitudinally, the peak malformation growth between 24 and 29 weeks of gestation was followed by a significant decrease in the absolute CVR to $0.43 \pm 0.07$ (Figure 3A, $p<0.0001$ ). Statistical significance with respect to the observed size progression and subsequent regression of these malformations was maintained after exclusion of maternal betamethasone patients $(p=0.02$ and $p<0.0001$, respectively).

\section{Correlation with postnatal outcome}

There were no cases of intrauterine demise. Gestational age at delivery was $38.9 \pm 0.28$ weeks (median weeks: 39.1 , IQR: 38.7-39.9). Two (5\%) fetuses, both with maximum CVRs greater than 2.0 after 29 weeks of gestation, had respiratory distress at birth and were managed with ventilator support and lobectomy in the neonatal period. There were no postnatal deaths.

Based on review of all postnatal chest CT scan reports obtained at our institution ( $n=35), 32(91.4 \%)$ children had an identifiable parenchymal abnormality of the lung consistent with their prenatal diagnosis. Three (8.6\%) had completely normal CT scans. The mean lesion maximum diameter was $2.71 \pm 0.28 \mathrm{~cm}$. The initial, mean, maximum, and final CVRs were significantly correlated with postnatal CT size dimensions 

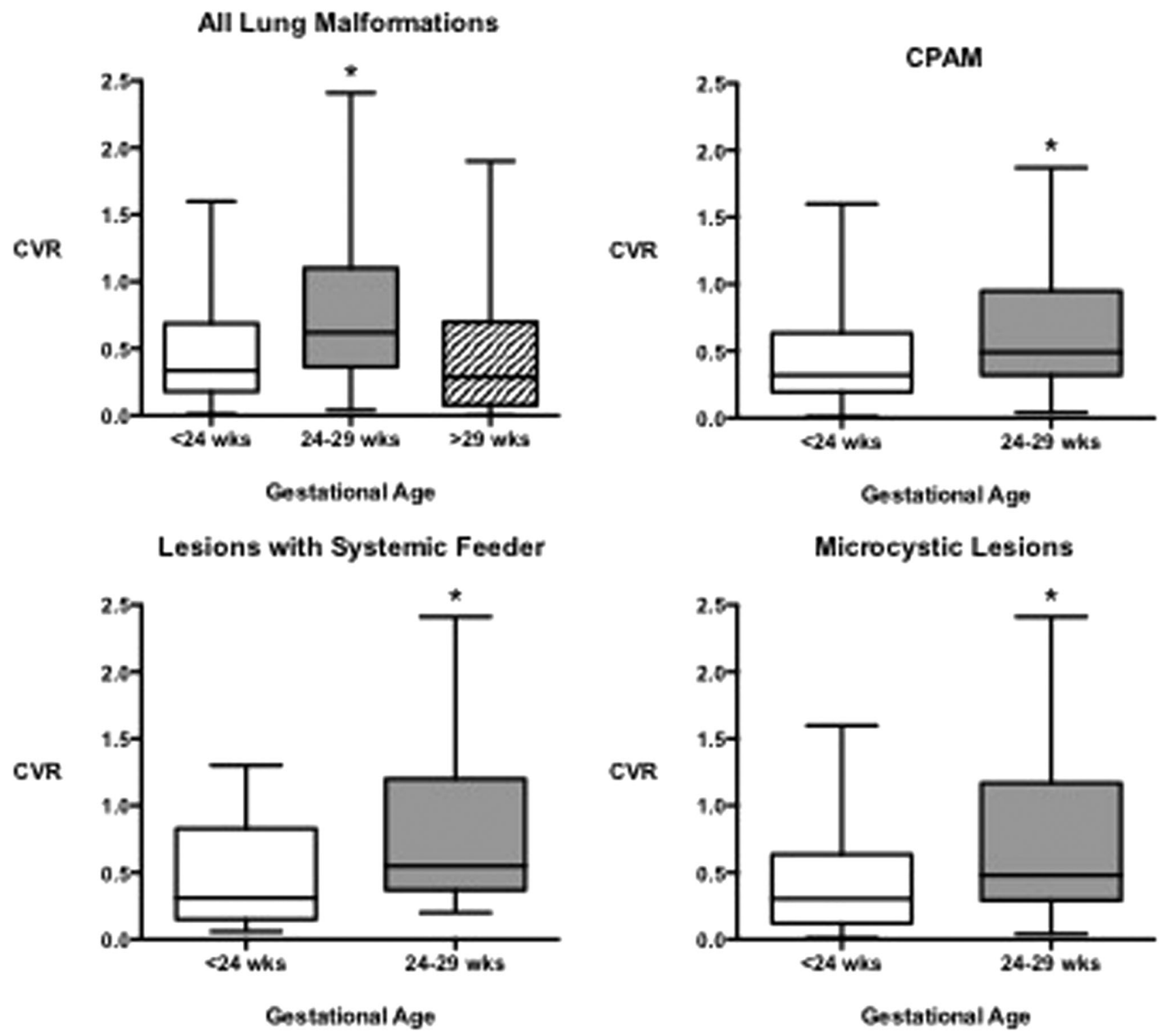

Figure 3 Box plots with whiskers from minimum to maximum representing absolute congenital pulmonary airway malformation volume ratio (CVR) data stratified by gestational age categories (the symbol '*' denotes $p<0.05$ compared with other groups). (A) All lung malformations measured before 24 weeks, between 24 and 29 weeks, and after 29 weeks of gestation. (B) Prenatal congenital pulmonary airway malformations (CPAM) masses measured before 24 weeks compared with 24-29 weeks of gestation. (C) Prenatal masses with a systemic feeder vessel measured before 24 weeks compared with 24-29 weeks of gestation. (D) Prenatal microcystic masses measured before 24 weeks compared with 24-29 weeks of gestation

(in $\mathrm{cm}$ ) based on linear regression analyses $(p=0.0016,<0.0001$, 0.0002 , and 0.0034, respectively). Of all CVR measurements, the mean CVR for a given fetus was the most significant and showed the strongest correlation with postnatal size by CT imaging ( $r=0.66$, Figure $4 \mathrm{~A}$ ).

Lung resection was performed in $26(65 \%)$ children at $6.4 \pm 0.67$ months of age (median months: 6.0, IQR: 4.9-8.0). Pathological evaluation of specimens revealed that nine (34.6\%) were CPAMs, five (19.2\%) were BPS, and nine (34.6\%) were hybrid lesions with features of both CPAM and BPS. Other pathology included a bronchogenic cyst in combination with congenital lobar emphysema $(n=1,3.8 \%)$ and segmental emphysema/bronchial atresia ( $n=2,7.7 \%)$. The mean CVR was significantly associated with an increased likelihood of postnatal lung surgery (Figure 4B, $p=0.0011$ ). Among those undergoing surgical resection, the mean CVR was $0.80 \pm 0.10$. Among those managed non-operatively, the mean CVR was $0.33 \pm 0.06$.

\section{DISCUSSION}

In this study, we used the CVR to evaluate the ultrasound growth patterns of prenatal lung masses using a large, prospectively collected database. Much like the lung-head ratio, $^{21}$ an ultrasound-derived parameter used to predict outcomes in fetal congenital diaphragmatic hernia, our group hypothesized that the relative size of lung malformations, as measured by the CVR, may be variable depending on the gestational age and therefore has implications for prenatal management and postnatal outcome. Using a systematic 


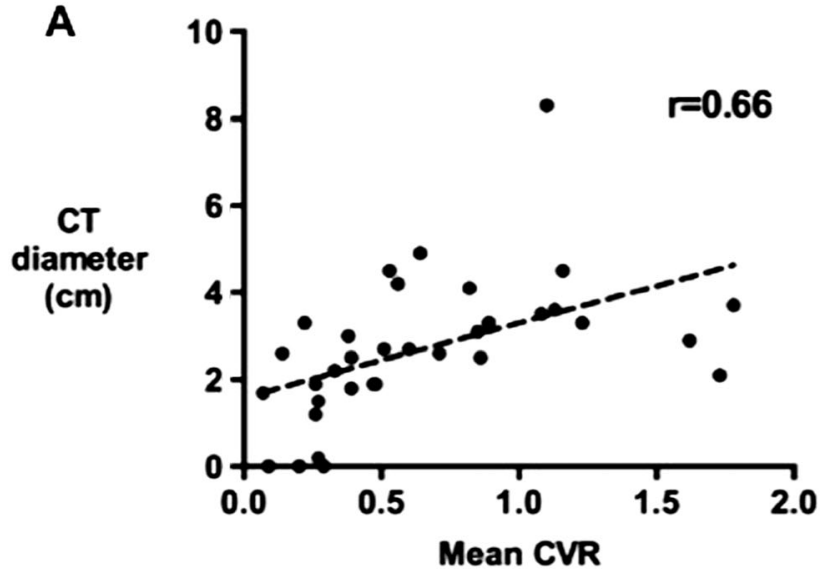

B

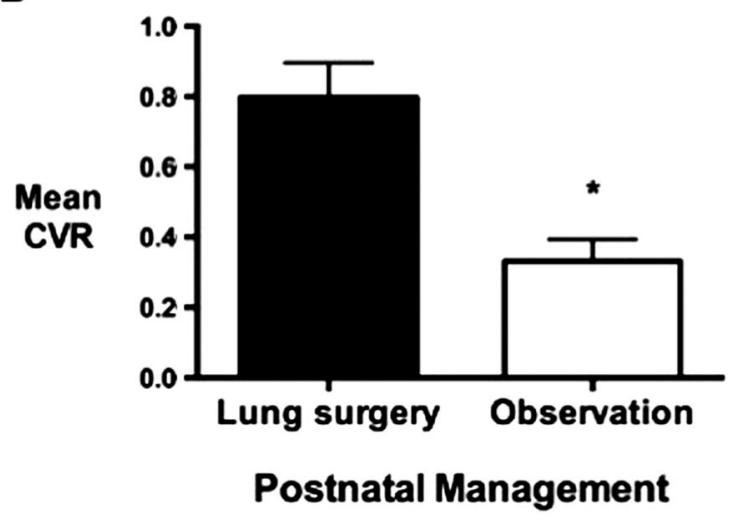

Figure 4 Relationship between prenatal congenital pulmonary airway malformation volume ratio (CVR) and postnatal outcome measures. (A) XY scatter plot showing relationship between mean CVR and postnatal dimensions by computed tomography (CT) scan (in $\mathrm{cm}$ ). (B) Column bar graph of the mean CVR and standard error of the mean in patients undergoing surgical resection versus observation (the symbol ${ }^{\prime * \prime}$ denotes $p<0.05$ )

approach that excluded late referrals and those with minimal follow-up, we found that the majority of malformations do not exhibit erratic growth. Instead, approximately two thirds of all lung lesions follow a predictable change in the CVR during the second trimester characterized by a modest but predictable increase in size following initial diagnosis. In these cases, the CVR tended to peak from 25 to 26 weeks of gestation. An approximately twofold increase in the CVR was seen in most lesions. This increase was consistent regardless of other sonographic features, including prenatal CPAM diagnosis, presence of a systemic feeding vessel, or microcystic appearance. On the other hand, roughly one third of lesions did not follow this anticipated growth profile and instead remained stable in size before regressing with advancing gestation. This highlights some inherent heterogeneity of growth patterns among prenatally diagnosed lung malformations.

It has been suggested that the fetus eventually 'grows around' the tumor as the thoracic volume begins to increase relative to overall somatic growth after 26 weeks of gestation. ${ }^{11,22}$ Our data suggest that after an initial size increase, what followed in most lesions was a proportionate decline in the CVR by 29 weeks of gestation. However, given that overall fetal lung volumes are known to increase relative to fetal head circumference during the third trimester, ${ }^{23}$ the observed decline in the size of CPAMs during this time period is unlikely to be explained solely by changes in overall somatic growth. Mass regression seems to correspond with the natural transition in fetal embryology between the canalicular and saccular stages of lung development. During this transition, investigators have hypothesized that an asynchrony in epithelial-mesenchymal interactions within CPAMs occurs, leading in alterations in cell proliferation relative to apoptosis. ${ }^{24}$ Because bronchial obstruction during early lung development may be an inciting event in the disease pathogenesis of lung malformations, ${ }^{25,26}$ some have postulated that alleviation of a transient bronchial mucous obstruction as the airways enlarge may also play a role in lesion size reduction. ${ }^{27}$

In this study, the mean CVR at initial presentation was 0.51, which is slightly lower than the mean initial CVR documented elsewhere ${ }^{9,13,28}$ but comparable with that reported in another study. ${ }^{8}$ Given that our lung lesions were, on average, small and all had an initial CVR less than or equal to 1.6, close longitudinal follow-up did not alter the prenatal management in most fetuses. Nonetheless, we believe serial ultrasound data were informative for several reasons. First, given the potential for longitudinal growth of some lesions, the gestational age when the CVR is measured and referred to tertiary care providers has relevance in terms of the need for close followup and accurate prognosis. We found that longitudinal tracking of the CVR also identified several patients deemed to be at increased risk for poorer outcomes. Second, we have found these data are useful for giving evidence-based information about the natural history of fetal lung lesions to expectant parents during initial prenatal counseling discussions. ${ }^{29}$ In approximately $90 \%$ of cases, the initial CVR will be less than 1.0. We would suggest serial ultrasounds every 2 weeks until evidence of CVR decline. In the majority of cases, either stable size or unequivocal regression will be seen by 29 weeks of gestation. Once the CVR has stabilized or begins to regress, ultrasound surveillance can be individualized with less frequent scanning.

A secondary objective of this study was to determine whether the CVR was an accurate measure of mass size when compared with postnatal CT scan, the current imaging gold standard. ${ }^{30,31}$ Despite changes observed in CVR growth over time, the initial, mean, maximum, and final CVRs were all significantly correlated with postnatal CT lesion size dimensions and likelihood for surgical resection. This data further validate the CVR as a reliable, quantitative tool that can be useful for predicting clinical outcomes after delivery. However, reliance on the final CVR in isolation may lead to a false negative diagnosis because some of these lesions can become isoechoic, particularly late in gestation. ${ }^{32}$ In all asymptomatic prenatally diagnosed cases, it remains imperative to image postnatally, preferrably by contrastenhanced CT scan, to further characterize any lesions if preemptive surgical resection is being considered. ${ }^{33,34}$

Although the current series using prospectively collected data has further elucidated the prenatal growth patterns 
oflung malformations, the study has some caveats and limitations. This is a single institution retrospective study based on prospectively collected data with stringent inclusion criteria. All four fetuses that developed hydrops were excluded because of late referral diagnosis $(n=2)$ or lack of serial measurements $(n=2)$, which was a common cause for exclusion before 2005. Furthermore, we acknowledge that many centers do not routinely measure $\mathrm{CVRs}^{8}$ and that obtaining an accurate CVR may involve a learning curve. Only seven macrocystic lesions were evaluated, and there is data to suggest that CPAMs with a dominant macrocyst may have unpredictable growth rates. ${ }^{13}$ The involvement of multiple maternal-fetal medicine providers may have allowed for increased measurement error. Additionally, we studied the full spectrum of lung malformations rather than restricting our analysis to pathologically confirmed CPAM lesions. While greater homogeneity of lung pathology would certainly be ideal, there is a high discordance between prenatal and postnatal diagnosis, particularly with respect to systemic feeding vessels. Interestingly, the current study also shows that BPS and other lesions have similar growth profiles compared with CPAMs. We therefore find that such pathologic distinctions are becoming increasingly irrelevant from a fetal diagnosis and management perspective. Finally, while many pediatric surgeons advocate for early surgical resection for most asymptomatic lung malformations given their long-term risk of complications, the threshold for surgery is specific to each institution and continues to evolve. $^{35-37}$

\section{ACKNOWLEDGEMENTS}

The authors wish to thank the Center for Statistical Consultation and Research (CSCAR) at the University of Michigan for providing their statistical expertise.

\section{WHAT'S ALREADY KNOWN ABOUT THE TOPIC?}

- The congenital pulmonary airway malformation volume ratio (CVR) is a common prenatal ultrasound measure of relative mass size in fetuses with lung malformations.

- The initial CVR and maximum CVR have been shown to be predictive of hydrops and neonatal respiratory compromise, respectively.

\section{WHAT DOES THIS STUDY ADD?}

- Gestational age is important when interpreting CVR measurements because two thirds of lesions increase in size at 25-26 weeks before spontaneous involution occurs.

- The mean CVR correlates with size measured by postnatal computed tomography scan

\section{REFERENCES}

1. Adzick NS. Management of fetal lung lesions. Clin Perinatol 2009;36:363-76 x.

2. Lima JS, Camargos PA, Aguiar RA, et al. Pre and perinatal aspects of congenital cystic adenomatoid malformation of the lung. J Matern Fetal Neonatal Med 2014;27:228-32.

3. Ehrenberg-Buchner S, Stapf AM, Berman DR, et al. Fetal lung lesions: can we start to breathe easier? Am J Obstet Gynecol 2013;208(151):e1-e7.

4. Adzick NS, Harrison MR, Glick PL, et al. Fetal cystic adenomatoid malformation: prenatal diagnosis and natural history. J Pediatr Surg 1985;20:483-8

5. Adzick NS, Harrison MR, Crombleholme TM, et al. Fetal lung lesions: management and outcome. Am J Obstet Gynecol 1998;179:884-9.

6. Miller JA, Corteville JE, Langer JC. Congenital cystic adenomatoid malformation in the fetus: natural history and predictors of outcome. J Pediatr Surg 1996;31:805-8.

7. Davenport M, Warne SA, Cacciaguerra S, et al. Current outcome of antenally diagnosed cystic lung disease. J Pediatr Surg 2004;39:549-56.

8. Ruchonnet-Metrailler I, Leroy-Terquem E, Stirnemann J, et al. Neonatal outcomes of prenatally diagnosed congenital pulmonary malformations. Pediatrics 2014;133:e1285-e1291.

9. Cass DL, Olutoye OO, Cassady CI, et al. Prenatal diagnosis and outcome of fetal lung masses. J Pediatr Surg 2011;46:292-298.

10. Hadchouel A, Benachi A, Revillon Y, et al. Factors associated with partial and complete regression of fetal lung lesions. Ultrasound Obstet Gynecol 2011;38:88-93.

11. Khalek N, Johnson MP. Management of prenatally diagnosed lung lesions. Semin Pediatr Surg 2013;22:24-9.

12. Laberge JM, Flageole H, Pugash D, et al. Outcome of the prenatally diagnosed congenital cystic adenomatoid lung malformation: a Canadian experience. Fetal Diagn Ther 2001;16:178-86.

13. Crombleholme TM, Coleman B, Hedrick H, et al. Cystic adenomatoid malformation volume ratio predicts outcome in prenatally diagnosed cystic adenomatoid malformation of the lung. J Pediatr Surg 2002;37:331-8.

14. Peranteau WH, Wilson RD, Liechty KW, et al. Effect of maternal betamethasone administration on prenatal congenital cystic adenomatoid malformation growth and fetal survival. Fetal Diagn Ther 2007;22:365-71.

15. Hedrick HL, Flake AW, Crombleholme TM, et al. The ex utero intrapartum therapy procedure for high-risk fetal lung lesions. J Pediatr Surg 2005;40:1038-1043 discussion 44.

16. Roggin KK, Breuer CK, Carr SR, et al. The unpredictable character of congenital cystic lung lesions. J Pediatr Surg 2000;35:801-5.

17. Platt LD, DeVore GR. In utero diagnosis of hydrops fetalis: ultrasound methods. Clin Perinatol 1982;9:627-36.

18. Cass DL, Olutoye OO, Ayres NA, et al. Defining hydrops and indications for open fetal surgery for fetuses with lung masses and vascular tumors. J Pediatr Surg 2012;47:40-5.

19. Haggerty JE, Smith EA, Kunisaki SM, et al. CT imaging of congenital lung lesions: effect of iterative reconstruction on diagnostic performance and radiation dose. Pediatr Radiol 2015;45:989-97.

20. Kunisaki SM, Powelson IA, Haydar B, et al. Thoracoscopic vs open lobectomy in infants and young children with congenital lung malformations. J Am Coll Surg 2014;218:261-70.

21. Jani J, Nicolaides KH, Keller RL, et al. Observed to expected lung area to head circumference ratio in the prediction of survival in fetuses with isolated diaphragmatic hernia. Ultrasound Obstet Gynecol 2007;30:67-71

22. Kunisaki SM, Barnewolt CE, Estroff JA, et al. Large fetal congenital cystic adenomatoid malformations: growth trends and patient survival. J Pediatr Surg 2007;42:404-10.

23. Peralta CF, Cavoretto P, Csapo B, et al. Assessment of lung area in normal fetuses at 12-32 weeks. Ultrasound Obstet Gynecol 2005;26:718-24.

24. Cass DL, Quinn TM, Yang EY, et al. Increased cell proliferation and decreased apoptosis characterize congenital cystic adenomatoid malformation of the lung. J Pediatr Surg 1998;33:1043-6 discussion 47.

25. Langston C. New concepts in the pathology of congenital lung malformations. Semin Pediatr Surg 2003;12:17-37. 
26. Kunisaki SM, Fauza DO, Nemes LP, et al. Bronchial atresia: the hidden pathology within a spectrum of prenatally diagnosed lung masses. J Pediatr Surg 2006;41:61-5 discussion 61-5.

27. Meizner I, Rosenak D. The vanishing fetal intrathoracic mass: consider an obstructing mucous plug. Ultrasound Obstet Gynecol 1995;5:275-7.

28. Yong PJ, Von Dadelszen P, Carpara D, et al. Prediction of pediatric outcome after prenatal diagnosis and expectant antenatal management of congenital cystic adenomatoid malformation. Fetal Diagn Ther 2012;31:94-102.

29. Aite L, Zaccara A, Trucchi A, et al. Is counselling for CCAM that difficult? Learning from parental experience. J Prenat Med 2011;5:65-8.

30. Epelman M, Kreiger PA, Servaes S, et al. Current imaging of prenatally diagnosed congenital lung lesions. Semin Ultrasound CT MR 2010;31:141-57.

31. Zeidan S, Gorincour G, Potier A, et al. Congenital lung malformation: evaluation of prenatal and postnatal radiological findings. Respirology 2009;14:1005-11.
32. Winters WD, Effmann EL, Nghiem HV, et al. Disappearing fetal lung masses: importance of postnatal imaging studies. Pediatr Radiol 1997;27:535-9.

33. Pumberger W, Hormann M, Deutinger J, et al. Longitudinal observation of antenatally detected congenital lung malformations (CLM): natural history, clinical outcome and long-term follow-up. Eur J Cardiothorac Surg 2003;24:703-11.

34. Kunisaki SM, Ehrenberg-Buchner S, Dillman JR, et al. Vanishing fetal lung malformations: prenatal sonographic characteristics and postnatal outcomes. J Pediatr Surg 2015;50:978-82.

35. Aziz D, Langer JC, Tuuha SE, et al. Perinatally diagnosed asymptomatic congenital cystic adenomatoid malformation: to resect or not? J Pediatr Surg 2004;39:329-34 discussion 29-34.

36. Wong A, Vieten D, Singh S, et al. Long-term outcome of asymptomatic patients with congenital cystic adenomatoid malformation. Pediatr Surg Int 2009;25:479-85.

37. $\mathrm{Ng} \mathrm{C}$, Stanwell J, Burge DM, et al. Conservative management of antenatally diagnosed cystic lung malformations. Arch Dis Child 2014;99:432-7. 